Una Reflexión del Grado de Empleabilidad de los Egresados UnNersitarios

Nuria Ceular Villamandos* José Maria Caridad y Ocerin** Federico Navarro Nieto ${ }^{* * *}$

\title{
RESUMEN:
}

Si bien uno de los problemas que afectan a todos los grupos de población es el desempleo, el objetivo del presente estudio es el análisis de esta situación entre los recién titulados. Asi, tal y como indica Corrales. et al (2003) una situación en la que la mayor parte de los jóvenes saliera del sistema educativo y encontrara sin dificultad un empleo sería ciertamente tranquilizadora, tanto para los jóvenes como para la sociedad en su conjunto, sin embargo, la realidad muestra que una parte importante de los jóvenes, tras salir de sistema educativo, se enfrenta a importantes dificultades para encontrar un empleo y, en muchos casos, acaba desempleado o inactivo. En este trabajo se presentan los resultados obtenidos del analisis del la situación laboral de los egresados de la Universidad de Córdoba de los cunsos académicos 2001/02 y 2003/04.

\section{Palabras Clave:}

Egresados. Inserción Laboral. Universidad. Empleo.

\section{ABSTRACT:}

Though one of the problems that affects the whole population is the unemployment, the aim of the present study is the analysis of this situation betueen newly university graduates. This way, as Corrales, et al (2003) indicates a situation in

- Prof ${ }^{2}$ Contratada Doctora de Organización de Empresas. Universidad de Córdoba - nuria.ceular@uco.es.

* Catedrático de Estadística, Econometría, e I.O. Universidad de Córdoba • ccjm@uco.es

*.* Catedrático de Derecho del Trabajo y de la Seguridad Social. Universidad de Córdoba • dt 1nanif@uco.es 
which most of the young people was going out of the educational system and they was finding without difficulty an employment it would be certainly encouraging, both for the young people and for the whole society, nevertbeless, the reality shows that an important part of the young people, after going out of the educational system, face important difficulties to find an employment and, in many cases, the person finishes unemployed or inactively.

In this work, we present the results obtained of the analysis of the labour situation of the graduated ones from the University of Córdoba of the academic courses 2001/02 and 2003/04.

KEY WORDS:

Graduated (gone away). Labour insertion. University. Employment.

\section{INTRODUCCIÓN}

En la actualidad, como han subrayado las instituciones de la UE, los sistemas de instrucción y formación cumplen en Europa un doble papel, de carácter social y económico. La educación constituye una parte integrante de la dimensión social europea, transmitiendo los valores de la solidaridad, de la igualdad de oportunidades y de la participación social ${ }^{1}$. Desde esta óptica, la educación superior es un factor relevante para incrementar las oportunidades de los individuos, favoreciendo su participación en la vida laboral y social, y por esta vía aumentando su bienestar social.

En concreto, se presenta el escenario real de los jóvenes titulados a partir de los datos de empleo, desempleo o inactividad de los jóvenes universitarios, así como las causas fundamentales de dicha situación. Con ello se pretende poner de manifiesto cual es la problemática real de éstos titulados al acceso tanto a su primer empleo como a otro empleo de cierta significación y reflexionar sobre las acciones y actividades a emprender para mejorar estos resultados.

Si bien uno de los problemas que afectan a todos los grupos de población es el desempleo, el objetivo del presente estudio es el análisis de esta situación entre los recién titulados. Así, tal y como indica Corrales, et al (2003) una situación en la que la mayor parte de los jóvenes saliera del sistema educativo y encontrara sin dificultad un empleo sería ciertamente tranquilizadora, tanto para los jóvenes como para la sociedad en su conjunto, sin embargo, la realidad muestra que una parte importante de los jóvenes, tras salir de sistema educativo, se enfrenta a importantes dificultades para encontrar un empleo y, en muchos casos, acaba desempleado o inactivo.

1. Consejo Europeo, Instrucción y formación 2.010: la urgencia de las reformas para el impulso de la estrategia de Lisboa, 3-3-2004 (documento del Consejo 6905/2004). 
El problema de la inserción laboral de los egresados ha generado numerosas investigaciones tanto en nuestro país como fuera de las fronteras. En ellos se refleja la preocupación por el proceso de transición al primer empleo de cierta significación para este colectivo. Entre ellos se destacan los trabajos de Aguilar, M.I. (2002), que a partir de datos de la Encuesta Sociodemográfica (INE) analiza la búsqueda del primer empleo de los universitarios; Dolado et al (2000); Alba Ramírez (1996), Cañada, et al (1998) que se basan en la información proporcionada por la Encuesta de Población Activa o Albert, et al (2003), Corrales y Rodríguez (2003) a partir del Módulo de Transición de la Educación al mercado laboral. Además de estas investigaciones a nivel nacional, y con el fin de analizar el proceso desde las propias universidades, se han generado numerosos estudios en ámbitos regionales más localizados, en los que al igual que la investigación que se presentan, se han diseñando encuestas específicas para abordar esta problemática. Entre estos destacamos a Salas Velasco (2003), Vidal, et al (2004), Martínez Martín (2002), Gil Jurado (1991) entre otros. Fuera de nuestro país, merece especial mención el trabajo de Jiménez, Sánchez y Campillo (2003) a partir de los datos de la conocida encuesta Cheers para los egresados de once países europeos y Japón.

Con el fin de complementar el estudio, en la tabla siguiente se presenta la evolución de la población activa en España en el período 1996-2005. Como se observa el número total de activos se sitúa a finales del 2005 en $20.956,1$ miles de personas, lo que implica un incremento del 16\% de la población activa existente en el año 2000. Del total, de población activa, se observa que el $58.8 \%$ es población masculina, con un total de $12.324,1$ miles de trabajadores activos. En lo que a población ocupada se refiere, en 2005 se alcanzó un máximo de 19.191,1 miles de individuos ocupados, de los que sólo el 40\% eran mujeres, y la población parada alcanza a un total de 1765,0 miles de individuos, de los que el $54,7 \%$ son mujeres. Por último, las cifras de inactivos crecen levemente en los últimos cinco años alcanzando un total del 17.533,5 miles de individuos de los que dos tercios aproximadamente son mujeres.

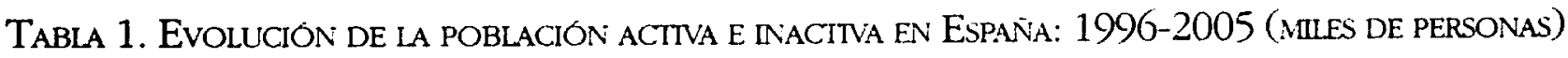

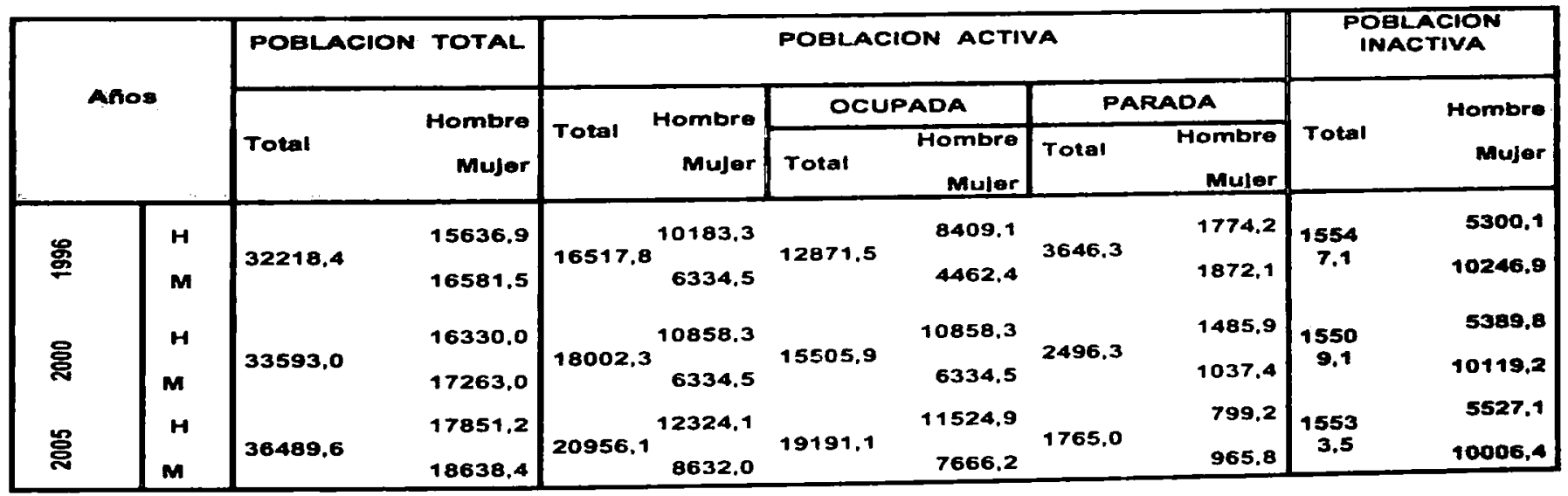

Fuente: MTAS (2005) y Elaboración Propia 
En cuanto a las tasas de actividad y de paro, se observa un incremento en aproximadamente $3,8 \%$ de la tasa de actividad en el periodo 2000-2005, llegando a alcanzar una tasa igual a 57,4 en 2005. Analizando los resultados por sexo, las tasa de actividad es del 69,0 para la población masculina y del 46,3 para la femenina.

TABla 2. Evolución de las tasas de actividad Y Paro EN EsPaña: 1996-2005

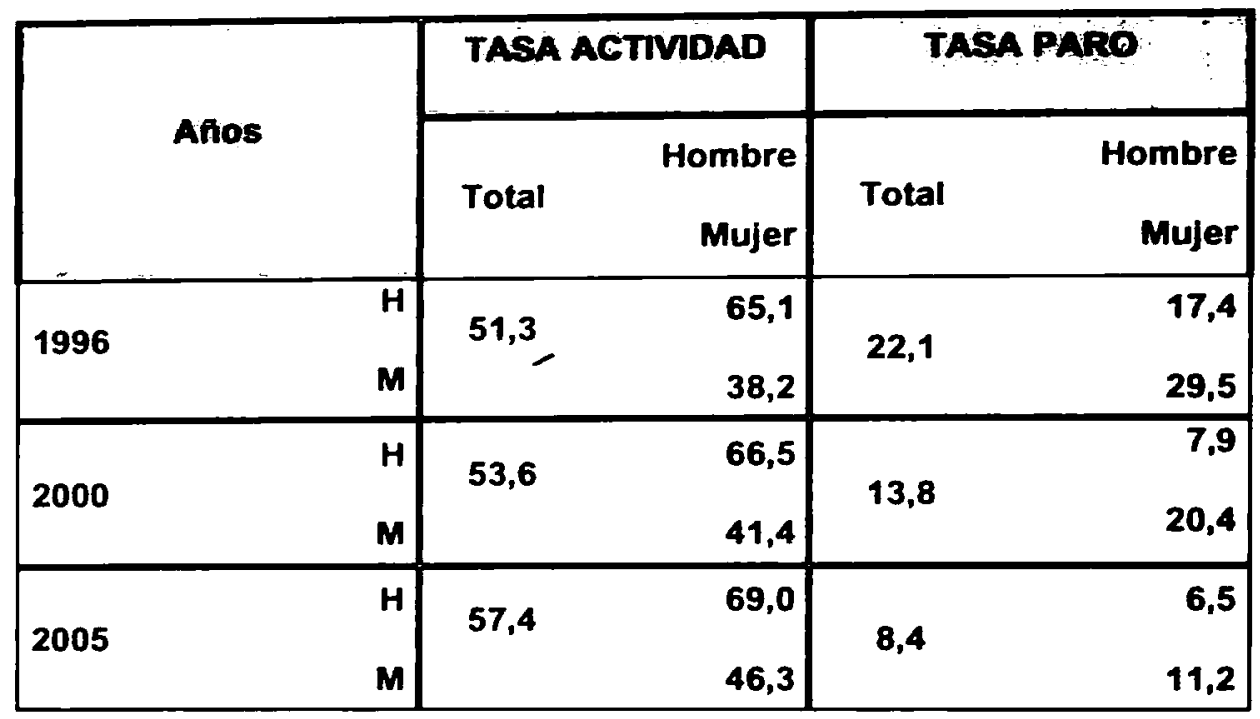

Fuente: MTAS (2005) y Elaboración Propia

No obstante lo anterior, la tasa de actividad se eleva cuando se analiza a la población con estudios universitarios. En la tabla siguiente se presentan los resultados de las tasas de actividad para la población universitaria española. Como se observa las tasas son superiores para los alumnos de segundo o tercer ciclo universitario, cuyo valor asciende al $83,5 \%$ sobre el total de población, frente al egresado con estudios de primer ciclo donde los valores son algo más reducidos $77,0 \%$.

TABLA 3. EvOlución de LAS TASAS DE ACTTVIDAD EN LOS UNIVERSTTARIOS ESPAÑOLES 2000-2005

\begin{tabular}{|c|ccc|ccc|}
\hline \multirow{2}{*}{ Años } & \multicolumn{3}{|c|}{ Primer Ciclo } & \multicolumn{3}{c|}{ Segundo Ciclo } \\
\cline { 2 - 7 } & Total & Hombre & Mujer & Total & Hombre & Mujer \\
\hline 2000 & 72.4 & 74.4 & 70.9 & 85.7 & 87.0 & 84.2 \\
\hline 2005 & 77.1 & 78.2 & 75.6 & 84.5 & 85.9 & 82.9 \\
\hline
\end{tabular}

Fuente: MTAS (2005) y Elaboración Propia 
Una vez reflejada la situación nacional se debe indicar que existen importantes diferencias con los resultados obtenidos para la comunidad andaluza. El mercado de trabajo andaluz presenta características diferenciales con respecto al resto de España, con una tasa de desempleo superior a la media española. Así a finales de 2005, cuando la tasa de desempleo nacional oscila en torno al 8,4, para el territorio andaluz supera el 13.8, llegando a alcanzar el 19.5 en el caso del desempleo femenino. Con esta situación se trata de diagnosticar cual es la realidad de la situación de los egresados una vez concluido su periplo universitario.

Por último, se debe aclarar que en la determinación de la población económicamente activa que figura en la EPA, se considera población económicamente inactiva a toda población que siendo mayor de dieciséis años y estando sin trabajo, no han buscado empleo. Frente a ello, la denominada población económicamente activa hace referencia al conjunto de personas mayores de dieciséis años que trabajan o están disponibles para trabajar y además realizan gestiones para incorporarse a la producción ${ }^{2}$.

\section{METODOLOGÍA UTILIZADA}

En el análisis de la situación actual de los egresados de la Universidad de Córdoba se determinaron un conjunto de objetivos variados y multidisciplinares, entre los que se destacan los siguientes: Obtener información sobre la situación en el mercado laboral de nuestros titulados. En concreto el estudio se ha desarrollado atendiendo a dos líneas de investigación complementarias. Por un lado, se analiza la situación de los titulados de recién incorporación al mercado laboral, el periodo de transición y el acceso al primer empleo de cierta significación. Por otro, se obtiene información de la situación de egresados de anterior incorporación, para estudiar el empleo más estable para que, sobre el nivel de adecuación de las exigencias cualitativas del mercado y de las competencias y habilidades adquiridas en el proceso de formación universitaria, plantearı estos titulados.

En el estudio se consideran dos poblaciones (independientes) constituidas por egresados de titulaciones oficiales de la universidad de Córdoba correspondientes a los cursos académicos 2001/02 y 2003/04. Así se ha conseguido analizar tanto a los titulados con al menos tres años de antigüedad en el mercado de trabajo, como al titulado de recién incorporación, (al menos un año).

Para el diseño muestral se consideró que cada población está dividida en estratos diseñados a partir de las titulaciones universitarias. A su vez dichos estratos han sido divididos en subestratos según el sexo del titulado, de manera que los respectivos tamaños muestrales quedaros finalmente determinados mediante muestreo estratificado. En concreto, se seleccionaron 750 individuos en ambas poblaciones estable-

2. Alcaide, $M$ et al (1996) 
ciéndose el tamaño muestral en cada estrato (y subestrato) mediante afijación proporcional. Teniendo en cuenta que las poblaciones son finitas, los tamaños muestrales obtenidos implican un error de $3 \%$ y $(2,91 \%$, para el primer y segundo colectivo respectivamente, en la estimación de proporciones para una confianza del 95,5\%.

TABLA 4. Ficha TÉCNICA DEL ESTUDiO

\begin{tabular}{|l|l|}
\hline POBLACIÓN OBJETO DE ESTUDIO & $\begin{array}{l}\text { EGRESADOS CURSO ACADEMICO 2001/02: 2434 individuos } \\
\text { EGRESADOS CURSO ACADEMICO 2003/04: 2062 individuos }\end{array}$ \\
\hline TAMAÑO DE LA MUESTRA & $\begin{array}{l}1500 \text { casos válidos (750 cada curso académico). } \\
\text { DISENADOS A PARTIR DE ESTRATOS DE TITULACIONES y } \\
\text { SUBESTRASTOS SEGUN SEXO }\end{array}$ \\
\hline $\begin{array}{l}\text { PROCEDIMIENTO DE RECOGIDA DE } \\
\text { INFORMACIÓN }\end{array}$ & $\begin{array}{l}\text { Cuestionario Telefónico + Protocolo recogida. } \\
\text { Cuestionario autoadministrado ubicado página web de la UCO } \\
\text { Cuestionario enviado por Correo Postal }\end{array}$ \\
\hline TIPO DE ENCUESTA & $\begin{array}{l}\text { Cuestionario semiestructurado de preguntas abiertas y } \\
\text { cerradas. (Escala 1 a } 5 \text { Likert fundamentalmente). }\end{array}$ \\
\hline Encuestas Psicologicas (Escala Satisfacción de Warr y Maslach \\
Burnout Inventory MBI)
\end{tabular}

Como se observa en el gráfico, la distribución de los egresados por macroáreas es similar en ambos colectivos. El porcentaje mayor corresponde en ambos casos a egresados de titulaciones pertenecientes a Ciencias Sociales y Jurídicas $(32,37 \%$ y $33,22 \%$ respectivamente). En segundo lugar se encuentran los egresados de titulaciones adscritas a Enseñanzas Técnicas $(23,13 \%$ y $24,59 \%)$ y Ciencias de la Salud $(20,75 \%$ y 18,23\%). Los egresados de titulaciones de Ciencias Experimentales (11,91\% y 13,19\%) y Humanidades $(11,83 \%$ y $10,77 \%)$ presentan menores valores. La distribución de los egresados por sexo y macroárea es similar en ambos colectivos. Se observa que el porcentaje de mujeres tituladas en el curso académico 2003/04 (57,13\%) aumenta levemente en relación al del curso 2001/02 (56,20\%).

Gráfico 1. Distribución POR macroáreas de CONOCIMIENTO
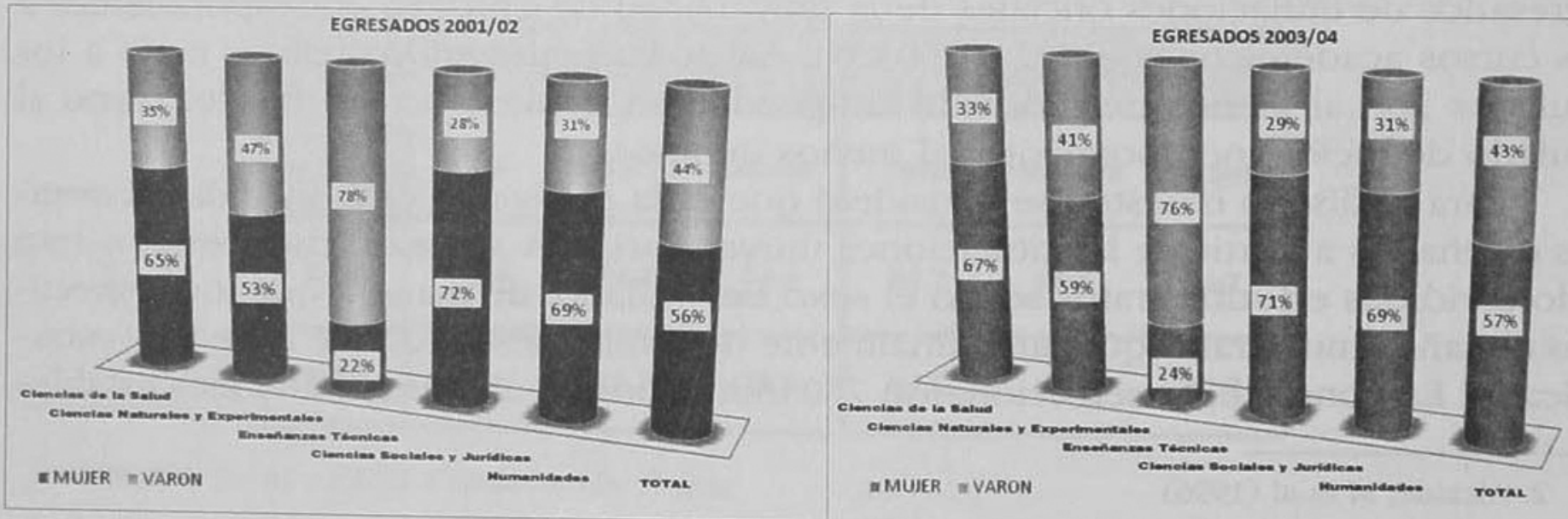
Para la recogida de información se elaboró un cuestionario en el que se presentan aspectos claves relacionados con el historial académico universitario, el proceso de inserción laboral y su situación actual en el mercado de trabajo. Una vez diseñado el cuestionario definitivo se procedió a la realización de la experiencia piloto. Durante una semana se pudo contrastar la tasa y el tiempo de respuesta, el protocolo de recogida de información, así como la necesidad de adecuar alguna pregunta cuyo enunciado pudiese resultar de difícil comprensión para el egresado.

Los resultados de la experiencia piloto se han utilizado para medir la fiabilidad interna de los cuatro conjuntos de ítems anteriores, para lo cual nos hemos basado en el denominado coeficiente alfa, más conocido como alfa de Cronbach. En este sentido, se observa en todos los casos, el valor de la alfa de Cronbach resulta superior a 0,7 , lo que permite afirmar que los grupos de ítems empleados para medir los aspectos citados tienen una buena fiabilidad interna, tanto mejor cuanto más próximo a 1.

Con el fin de garantizar la tasa de respuesta, el proceso de recogida de información finalmente utilizado se realiza por tres vías: Vía Telefónica: El encuestador, a partir del protocolo que se diseñó para el proceso, se presentaba al alumnado vía telefónica solicitando su participación en la investigación, dándole la posibilidad de comunicarse en otro momento si no estaba disponible en ese instante. A continuación se procedía a la realización de la encuesta y a su cumplimentación en soporte papel para minimizar el tiempo de respuesta y evitar errores de mecanización de manera automática sin posibilidad de comprobación futura. El tiempo medio de respuesta se aproximó a catorce minutos. En lo que a la jornada se refiere, indicar que como durante la mañana resultaba más difícil comunicar con el egresado, se procedió a desdoblar y permutar las jornadas de los encuestadores para poder alcanzar los objetivos propuestos. Vía telemática: Para facilitar la tasa de respuesta se facilita un portal en la Web de donde se presenta un cuestionario telemático cuya respuesta queda directamente vinculada a una base de datos, y cuya dirección se le proporciona a todo el alumno que esté interesado. Esta opción fue utilizada por un porcentaje muy reducido de egresados. Vía Postal: Como última opción se facilita la posibilidad de enviar por correo postal o electrónico el cuestionario, para su cumplimentación manual y posterior devolución vía postal. Esta última alternativa no fue elegida por ningún egresado.

\section{RESULTADOS}

En este apartado se describe la situación actual del egresado en lo que a su ocupación se refiere. Para su análisis, se distinguirá entre el egresado activo, tanto ocupado como parado por un lado egresado inactivo por otro. 


\subsection{El Egresado Activo}

La población egresada activa (suma del total de ocupados y de parados buscando empleo) de los egresados de 2001/02 y 2003/04 es del $83,8 \%$. Por sexo, la tasa es superior entre el colectivo masculino ( $87,9 \%$ frente al $80,6 \%$ femenino). Por macroáreas se observa que las Enseñanzas Técnicas y Ciencias de la Salud presentan los mayores indices de actividad (superiores al 90\%) frente a las titulaciones asociadas a Humanidades (que tan solo alcanzan un $66,8 \%$ ).

Tomando como referencia los egresados encuestados de 2001/02 y 2003/04 cabe deducir una ocupación del $66,8 \%$. Puede tomarse en consideración que de los estudios realizados para titulados de otras Universidades públicas españolas se deduce que la población ocupada entre titulados universitarios oscila entre el $55 \%$ y el 83\% (respecto de estos estudios, Informe CYD 2006).

En la distribución por sexo, la tasa de ocupación es superior entre los egresados masculinos. Por macroáreas de conocimiento, las titulaciones que presentan tasas de ocupación más elevadas corresponden a las asociadas a Enseñanzas Técnicas, Ciencias de la Salud y Ciencias Sociales y Jurídicas. Son más reducidas en Ciencias Experimentales y especialmente en Humanidades.

Tabla 5. Análisis de la Población Activa

\begin{tabular}{|c|c|c|c|c|c|}
\hline & \multirow{2}{*}{ Poblicián } & \multirow{2}{*}{ POBLACIÓN TOTAL } & \multicolumn{3}{|c|}{ PoBiofownom, } \\
\hline & & & TASA ACTIVIDAD & OCUPADOS & \% OCUPADOS \\
\hline 㺃 & $\begin{array}{l}\text { Hombres } \\
\text { Mujeres }\end{array}$ & $\begin{array}{l}655(43,6 \%) \\
845(56,3 \%)\end{array}$ & $\begin{array}{l}87,94 \% \\
80,59 \%\end{array}$ & $\begin{array}{l}492 \\
510\end{array}$ & $\begin{array}{l}49,10 \% \\
50,90 \%\end{array}$ \\
\hline 홍 & $\begin{array}{l}\text { Ciencias Salud } \\
\text { C. Experimentales } \\
\text { Enseñanzas Técnicas } \\
\text { Clencias Sociales } \\
\text { Humanidades }\end{array}$ & $\begin{array}{l}287(19,1 \%) \\
191(12,7 \%) \\
358(23,8 \%) \\
495(33,0 \%) \\
169(11,2 \%)\end{array}$ & $\begin{array}{l}90,94 \% \\
86,39 \% \\
91,90 \% \\
78,59 \% \\
66,86 \%\end{array}$ & $\begin{array}{l}218 \\
110 \\
309 \\
301 \\
64\end{array}$ & $\begin{array}{l}21,76 \% \\
10,98 \% \\
30,84 \% \\
30,04 \% \\
6,39 \%\end{array}$ \\
\hline 엉 & $\begin{array}{l}\text { Primer ciclo } \\
\text { Segundo ciclo }\end{array}$ & $\begin{array}{l}598(39,8 \%) \\
902(60,2 \%)\end{array}$ & $\begin{array}{l}87,29 \% \\
81,49 \%\end{array}$ & $\begin{array}{l}416 \\
586\end{array}$ & $\begin{array}{l}41,52 \% \\
58.48 \%\end{array}$ \\
\hline$\frac{1}{8}$ & $\begin{array}{l}\text { Cohorte 2001/02 } \\
\text { Cohorte 2003/04 }\end{array}$ & $\begin{array}{l}750(50,0 \%) \\
750(50,0 \%)\end{array}$ & $\begin{array}{l}87,73 \% \\
79,87 \%\end{array}$ & $\begin{array}{l}539 \\
463\end{array}$ & $\begin{array}{l}53,79 \% \\
46,21 \%\end{array}$ \\
\hline & TOTAL UNIVERSIDAD & 1500 & $83.8 \%$ & 1002 & $100,00 \%$ \\
\hline
\end{tabular}




\section{Población OCUPADA}

Según se desprende de la tabla siguiente, el porcentaje de alumnos empleados es, para los que finalizaron sus estudios en el curso académico 2001/02 y 2003/04, de un $71.9 \%$ y un $66.8 \%$ respectivamente. Se observa a partir de los resultados que existen diferencias significativas en la proporción de parados en cada grupo $(p$-valor $=0$ ), y de la información de la tabla se concluye que dicha proporción es superior en los egresados de 2003/04.

Además, existen diferencias significativas en la proporción de hombres y mujeres que actualmente están en paro ( $\mathrm{p}$-valor $=0$ ), y de la información de la tabla se concluye que dicha proporción es superior en el caso de las mujeres.

Tabla 6. Análisis de la Población Empleada

\begin{tabular}{|c|c|c|c|c|c|c|c|}
\hline EGRESADOS & SI TRABAJAN & NO TRABAJAN & TOTAL & SEXO & SI TRABAJAN & $\begin{array}{c}\text { NO } \\
\text { TRABAJAN }\end{array}$ & TOTAL \\
\hline $2001 / 2002$ & $71,9 \%$ & $28,1 \%$ & 750 & Hombre & $75,1 \%$ & $24,9 \%$ & 655 \\
\hline $2003 / 2004$ & $61,7 \%$ & $38,3 \%$ & 750 & Mujer & $60,4 \%$ & $39,6 \%$ & 845 \\
\hline Total & $66,8 \%$ & $33,2 \%$ & 1500 & Total & $66,8 \%$ & $33,2 \%$ & 1500 \\
\hline
\end{tabular}

La duración media del alumno en su trabajo actual es de aproximadamente 30,2 y 25,5 meses para sendos colectivos respectivamente. Según el test $\mathrm{T}$ de comparación de medias (asumiendo varianzas iguales según se desprende del test de Levene) los dos grupos de egresados no presentan diferencias significativas al $5 \%(\mathrm{p}$-lim $=0,081$ ) en lo que se refiere a la duración en el trabajo actual. En la tabla 4 se presentan los resultados obtenidos en el análisis de la duración del empleo por sexo.

Por otro lado, los resultados del test $\mathrm{T}$ de comparación de medias permiten afirmar que, por término medio, el número de empleos desempeñados por los individuos hasta encontrar el actual es superior en el grupo de egresados de 2003/04 ( $\mathrm{p}$-lim=0), lo que parece indicar que existe una tendencia a la rotación mayor en el empleo actual.

Tabla 7. Duración en el último empleo (meses) por sexo

\begin{tabular}{|c|c|c|c|c|c|c|c|c|}
\hline \multicolumn{2}{|c|}{ Sexo } & Media & Mediana & Moda & $\begin{array}{c}\text { Desviación } \\
\text { trp. }\end{array}$ & $\begin{array}{c}\text { Percentil } \\
25\end{array}$ & $\begin{array}{c}\text { Percentil } \\
75\end{array}$ & Total \\
\hline Egresados & Hombre & 34,26 & 24,00 & 36,00 & 39,02 & 12,00 & 42,00 & 262 \\
\hline $2001 / 02$ & Mujer & 26,35 & 24,00 & 36,00 & 31,54 & 8,50 & 36,00 & 277 \\
\hline & TOTAL & 30,19 & 24,00 & 36,00 & 35,56 & 12,00 & 36,00 & 539 \\
\hline Egresados & Hombre & 24,23 & 14,00 & 24,00 & 42,32 & 6,00 & 24,00 & 230 \\
\hline $2003 / 04$ & Mujer & 26,67 & 12,00 & 12,00 & 56,64 & 6,00 & 24,00 & 233 \\
\hline & TOTAL & 25,46 & 12,00 & 24,00 & 50,00 & 6,00 & 24,00 & 463 \\
\hline
\end{tabular}


En relación al periodo de transición o tiempo medio en encontrar su empleo actual, los resultados del test $\mathrm{T}$ de comparación de medias permiten afirmar que el tiempo medio que tardaron los individuos en encontrar el trabajo actual es superior en el grupo de egresados de 2001/02 (p-valor $=0$ ). Dentro de cada grupo de egresados la comparación anterior por sexos mediante el citado test $\mathrm{T}$ lleva a la misma conclusión: las mujeres tardan, por término medio, más tiempo que los hombres en encontrar su trabajo actual.

Tabla 8. Duración en la búsqueda del empleo actual.

\begin{tabular}{|lccccccc|}
\hline & Media & Mediana & Moda & $\begin{array}{c}\text { Desviación } \\
\text { tip. }\end{array}$ & $\begin{array}{c}\text { Percentil } \\
25\end{array}$ & $\begin{array}{c}\text { Percenfi } \\
75\end{array}$ & Total \\
\hline Egresados 2001/02 & 9,58 & 5,00 & $-1,00$ & 11,78 & 1,00 & 12,00 & 538 \\
\hline Egresados 2003/04 & 5,16 & 2,00 & 1,00 & 6,69 & 1,00 & 6,00 & 463 \\
\hline
\end{tabular}

Los resultados del test $\mathrm{T}$, permiten afirmar que el tiempo medio que tardaron los individuos en encontrar el trabajo actual es superior en el grupo de egresados de 2001/02 (p-valor $=0$ ). Dentro de cada grupo de egresados la comparación anterior por sexos mediante el citado test $\mathrm{T}$ lleva a la misma conclusión: las mujeres tardan, por término medio, más tiempo que los hombres en encontrar su trabajo actual

En cuanto a la rotación en empleos anteriores se permite afirmar que, por término medio, el número de empleos desempeñados por los individuos hasta encontrar el actual es superior en el grupo de egresados de 2003/04 ( $p$-valor $=0$ ), lo que parece indicar que existe una tendencia a la rotación de empleo en la actualidad. Además, no se detectan diferencias significativas entre hombres y mujeres en relación al número de empleos anteriores incluido el actual.

TABLA 9. EMPLEOS ANTERIORES AL ACtUAL.

\begin{tabular}{|lccccccc|}
\hline & Media & Mediana & Moda & $\begin{array}{c}\text { Desviación } \\
\text { tip }\end{array}$ & $\begin{array}{c}\text { Percentil } \\
\text { Eercentil }\end{array}$ & Tolal \\
\hline Egresados 2001/02 & 1,17 & 1,00 & 0,00 & 1,61 & 0,00 & 2,00 & 534 \\
\hline Egresados 2003/04 & 1,86 & 1,00 & 1,00 & 2,43 & 1,00 & 2,00 & 338 \\
\hline
\end{tabular}

En lo que se refiere a la prestación de servicios, el grueso de los titulados se consideran asalariados por cuenta ajena (un 31,5\% indefinido y un $37,3 \%$ con contrato temporal, un $3.2 \%$ en prácticas). Un $2,2 \%$ indica que o bien no tiene contrato o desconoce la existencia del mismo. El empleo por cuenta propia ocupa al 10,1\% de 
los titulados; y el empleo como funcionarios públicos el 15,8\%. De los estudios realizados para titulados de otras Universidades públicas españolas se deduce que la contratación temporal entre titulados universitarios oscila entre el 40 y el 56\% (respecto de estos estudios, Informe CYD 2006).

En cuanto a la duración del contrato temporal, los resultados del test chi-cuadrado de homogeneidad indican que, en el conjunto total de egresados, la distribución de hombres y mujeres con contrato temporal no es la misma en todas las macroáreas, siendo Ciencias Sociales y Jurídicas y Ciencias de la Salud donde se observa mayor utilización de contratos de este tipo. Esta misma conclusión se mantiene cuando se repite la comparación dentro de cada grupo de egresados. Los resultados del test $T$ de comparación de medias permiten afirmar que, para los individuos que tienen contrato temporal, no existen diferencias en la duración media de dichos contratos entre hombres y mujeres en ambos grupos de egresados.

Tabla 10. Duracion del contrato temporal

\begin{tabular}{|c|c|c|c|c|c|c|c|c|}
\hline \multirow{2}{*}{$\because$ MACROAREA } & \multicolumn{4}{|c|}{ Egresados 2001/02 } & \multicolumn{4}{|c|}{ Egresados 2003/04 } \\
\hline & Media & Medlana & Moda & $\begin{array}{c}\text { Desviación } \\
\text { tip. }\end{array}$ & Media & Mediana & Moda & $\begin{array}{c}\text { Desviación } \\
\text { típ. }\end{array}$ \\
\hline Clenclas de la Salud & 10,32 & 9,00 & 12,00 & 8,709 & 16,65 & 12,00 & 12,00 & 15,338 \\
\hline Ciencias Experimentales & 24,43 & 12,00 & 12,00 & 18,30 & 8,79 & 6,00 & 6,00 & 8,451 \\
\hline Enseñanzas Técnicas & 12,56 & 12,00 & 12,00 & 11,33 & 14,57 & 12,00 & 12,00 & 9,63 \\
\hline Clencias Sociales y & 7,35 & 6,00 & 6,00 & 5,213 & 8,88 & 9,0 & 9,00 & 7,857 \\
\hline Humanidades & 8,50 & 6,00 & 6,00 & 4,165 & 5,75 & 6,00 & 6,00 & 0,5 \\
\hline
\end{tabular}

Se presentan los resultados según el colectivo de egresados analizados. Para el colectivo 2001/02, el sector económico de mayor empleo es el correspondiente a Actividades Sanitarias y Veterinarias, con un $22,3 \%$ del titulado.- un $88,1 \%$ del empleo de los alumnos de Ciencias de la Salud-. Le sigue Educación con un 18,4\% de universitarios que proceden fundamentalmente de titulaciones relacionadas con las Ciencias Sociales y Jurídicas (39,2\%). Para el 2003/04 los sectores de mayor empleo siguen siendo los mismos. Por sectores económicos, y tomando en consideración conjuntamente los cursos académicos 2001/02 y 2003/04, el mayor empleo corresponde a actividades sanitarias y veterinarias, educación, Administraciones Públicas, y el sector de construcción. Por el contrario, hostelería, transportes, intermediación financiera, o comercio, presentan indicadores bastante más bajos. Considerando el tamaño de la empresa el 51,2\% de los egresados asalariados trabaja en PYMES (hasta 50 trabajadores de plantilla).

En cuanto al tamaño de la empresa se destaca el alto porcentaje de asalariados en empresas de más de 500 trabajadores de plantilla (el 29,1\%), lo que encuentra cierta explicación si se tiene en cuenta que dicho colectivo se concentra 
en Ciencias de la Salud y Enseñanzas Técnicas (el 74,7\% de los titulados que trabajan en empresas de estas dimensiones).

TABla 11. TAMaÑo DE LA EMPRESA

\begin{tabular}{|c|c|c|c|c|c|c|}
\hline Ne Trabajadores & $\begin{array}{l}\text { Cienclas de } \\
\text { la Salud }\end{array}$ & $\begin{array}{c}\text { Ciencias } \\
\text { Experimentales }\end{array}$ & $\begin{array}{c}\text { Enseffanzas } \\
\text { Técnicas }\end{array}$ & $\begin{array}{l}\text { Clencias } \\
\text { Sociales y } \\
\text { Juridicas }\end{array}$ & $\begin{array}{c}\text { Humanidade } \\
\mathrm{s}\end{array}$ & Tohnt \\
\hline Menos de 5 & $29,4 \%$ & $6,7 \%$ & $19,0 \%$ & $38,0 \%$ & $6,7 \%$ & 163 \\
\hline Entre 6 y 20 & $14,9 \%$ & $9,4 \%$ & $22,7 \%$ & $45,9 \%$ & $7,2 \%$ & 181 \\
\hline Entre 21 y 50 & $9,8 \%$ & $15,9 \%$ & $18,9 \%$ & $49,4 \%$ & $6,1 \%$ & 164 \\
\hline Entre 51 y 100 & $22,8 \%$ & $13,9 \%$ & $31,6 \%$ & $22,8 \%$ & $8,9 \%$ & 79 \\
\hline Entre 101 y 250 & $11,5 \%$ & $13,5 \%$ & $40,4 \%$ & $26,9 \%$ & $7,7 \%$ & 52 \\
\hline Entre 251 y 500 & $19,0 \%$ & $3,2 \%$ & $46,0 \%$ & $20,6 \%$ & $11,1 \%$ & 63 \\
\hline Más de $\mathbf{5 0 0}$ & $30,4 \%$ & $10,7 \%$ & $44,3 \%$ & $10,4 \%$ & $4,2 \%$ & 289 \\
\hline TOTAL & $21,7 \%$ & $10,6 \%$ & $30,9 \%$ & $30,4 \%$ & $6,5 \%$ & 991 \\
\hline
\end{tabular}

En nuestro estudio se aprecia que una mayoría de egresados dispone de unos ingresos que se sitúan en un intervalo comprendido entre los 9.000 y 18.000 euros anuales (el 55,5\%); si bien cabe advertir que otro segmento relevante se sitúa entre los 27.000 y los 36.000 euros (41\%). Son ilustrativas las diferencias apreciables en los niveles adquisitivos de los egresados si consideramos las macroáreas o titulación de origen. Así, por ejemplo, mientras las Enseñanzas Técnicas y las Ciencias de la Salud presentan un nivel salarial superior, las Ciencias Jurídicas y las Humanidades son retribuidas en menor cuantía. De la información estadística referida a las percepciones salariales de titulados y tituladas se deduce que la remuneración media de los varones está por encima de la percibida por las mujeres. No obstante, el dato no permite ir más allá en el análisis al no disponerse de datos sobre modalidades contractuales y categorías profesionales.

\section{Población Parada}

El total de egresados que en la actualidad están sin empleo suponen un tercio de los egresados encuestados (498). De éstos, la mitad son parados, es decir realizando egresados desocupados realizando actualmente una búsqueda activa de empleo 255 titulados, que suponen un $17 \%$ del total de los egresados. En cuanto a la inactividad, se observa que ésta alcanza a un 16\% de los egresados encuestados, siendo 243 egresados desempleados los que indicaron no estar realizando está búsqueda de empleo. 
Tabla 12. Análisis de la Población Parada

\begin{tabular}{|c|c|c|c|c|c|}
\hline \multicolumn{2}{|c|}{ POBLACION } & \multirow{2}{*}{$\begin{array}{l}\text { POBLACIÓN } \\
\text { TOTAL } \\
655(43,6 \%) \\
845(56,3 \%)\end{array}$} & \multicolumn{2}{|c|}{$\begin{array}{l}\text { POBLACIÓN } \\
\text { INACTIVA }\end{array}$} & \multirow{2}{*}{$\begin{array}{r}\begin{array}{r}\text { TASA } \\
\text { INACTIVIDAD } \\
12,06 \% \\
19,41 \%\end{array} \\
\end{array}$} \\
\hline 量 & $\begin{array}{l}\text { Hombres } \\
\text { Mujeres } \\
\end{array}$ & & $\begin{array}{l}79 \\
164\end{array}$ & $\begin{array}{l}32,50 \% \\
67,50 \%\end{array}$ & \\
\hline$\frac{\mathbb{2}}{\frac{\pi}{2}}$ & $\begin{array}{l}\text { Ciencias Salud } \\
\text { C. Experimentales } \\
\text { Enseñanzas Técnicas } \\
\text { Ciencias Sociales } \\
\text { Humanidades }\end{array}$ & $\begin{array}{l}287(19,1 \%) \\
191(12,7 \%) \\
358(23,8 \%) \\
495(33,0 \%) \\
169(11,2 \%)\end{array}$ & $\begin{array}{l}26 \\
26 \\
29 \\
106 \\
56\end{array}$ & \begin{tabular}{l|}
$10,70 \%$ \\
$10,70 \%$ \\
$11,93 \%$ \\
$43,62 \%$ \\
$23,05 \%$ \\
\end{tabular} & \begin{tabular}{|l|}
$9,06 \%$ \\
$13,61 \%$ \\
$8,10 \%$ \\
$21,41 \%$ \\
$33,14 \%$ \\
\end{tabular} \\
\hline 원 & $\begin{array}{l}\text { Primer ciclo } \\
\text { Segundo ciclo }\end{array}$ & $\begin{array}{l}598(39,8 \%) \\
902(60,2 \%)\end{array}$ & $\begin{array}{l}76 \\
167\end{array}$ & $\begin{array}{l}31,27 \% \\
68,73 \%\end{array}$ & $\begin{array}{l}12,71 \% \\
18,51 \%\end{array}$ \\
\hline س & $\begin{array}{l}\text { Cohorte 2001/02 } \\
\text { Cohorte 2003/04 }\end{array}$ & $\begin{array}{l}750(50,0 \%) \\
750(50,0 \%)\end{array}$ & $\begin{array}{l}92 \\
151\end{array}$ & $\begin{array}{l}37,86 \% \\
62,14 \%\end{array}$ & $\begin{array}{l}12,27 \% \\
20,13 \%\end{array}$ \\
\hline & OTAL UNIVERSIDAD & 1500 & 243 & $\cdots$ & $16,1 \%$ \\
\hline
\end{tabular}

Se destaca que tanto el porcentaje de desempleo como de inactividad es superior entre el colectivo femenino que el masculino. En ambos periodos el número de mujeres desocupadas e inactivas es mayor que el de los hombres. A continuación se desarrollan sendos colectivos -parados e inactivos- de manera diferenciada.

Tal y como se ha indicado anteriormente, se observa que la proporción de parados es superior entre el colectivo femenino que entre el masculino (171 egresadas frente a 84 egresados en demandando empleo), lo que supone que dos de cada tres egresados en esa situación son mujeres. Este dato refleja la situación más desfavorecida de la mujer titulada en la universidad, que si bien supera en proporción al egresado varón,- 56\% de los individuos son mujeres-, esta proporción no explica por sí misma el comportamiento del mercado laboral. La tasa de paro el colectivo femenino supera el $25 \%$ frente a la del masculino, considerablemente inferior, un $14,5 \%$.

Por macroáreas, las tasas de paro son diferentes. En las titulaciones asociadas a Humanidades y Ciencias Experimentales se alcanzan valores superiores al $43 \%$, y $33 \%$ respectivamente, frente a Enseñanzas Técnicas donde dicha tasa se reduce al 
6\%. En cuanto al ciclo correspondiente, las tasas de paro son similares para las titulaciones de primer o de segundo ciclo. Por último, según los cohortes de edad establecidos se observa que para los egresados de recién incorporación la tasa de paro aumenta, situación lógica por su recién incorporación al mercado laboral.

En la distribución de los egresados parados según la calificación obtenida en el expediente universitario, se observa que sólo el 14.8\% tiene un expediente de notable o sobresaliente, siendo aprobado el obtenido por el $85.09 \%$ restante. Analizando la distribución del expediente se observa que el expediente no explica la situación de desempleo/empleo del egresado.

Respecto al tiempo que llevan buscando empleo, para los egresados desempleados del curso académico 2001/02 y 2003/04 se destaca que un $36,13 \%$ y un $24,26 \%$ respectivamente afirman llevar más de dos años realizando una búsqueda activa de empleo. Entre las causas se destaca que de los egresados de los cursos $2001 / 02$ y $2003 / 04$, el $34 \%$ y el $29 \%$ afirman haber estado preparando oposiciones, en tanto que siempre ha permanecido desempleado en torno a un $10 \%$. Los egresados en 2001/02 manifiestan haber tenido trabajos temporales relacionados con sus estudios en un $29,4 \%$ mientras que los que finalizaron en $2003 / 04$ son el $24,2 \%$.

La principal causa de desempleo según los egresados de 2001/02 y 2003/04 es el mercado laboral. Así lo afirman el $63 \%$ y el $57,4 \%$ respectivamente. Como causa secundaria encontramos que la titulación es inadecuada para los trabajos que se ofrecen u otras causas.

Entre los encuestados desempleados de los cursos 2001/02 y 2003/04, el 34\% y el $29 \%$ afirman haber estado preparando oposiciones, en tanto que siempre ha permanecido desempleado en torno a un 10\%. Los egresados en 2001/02 manifiestan haber tenido trabajos temporales relacionados con sus estudios en un $29,4 \%$ mientras que los que finalizaron en $2003 / 04$ son el $24,2 \%$. Por el contrario, analizando los trabajos temporales no relacionados con los estudios, el $8 \%$ de los graduados en 2001/02 afirma haber realizado alguno de ellos, en tanto que los que finalizaron posteriormente ascienden hasta el 13\%. Entre el $11 \%$ y el $13 \%$ aproximadamente de los titulados han continuado estudiando.

La mayoría de los egresados no percibe prestación por desempleo. En el caso de los que finalizaron sus estudios en 2003/04 el porcentaje alcanza el 92,6\% del total desempleado. Se aplica el test chi-cuadrado de independencia en tabla de contingencia para comparar las proporciones de individuos desempleados que perciben prestación en cada uno de los grupos de egresados considerados. Los resultados indican que no existen diferencias significativas en la proporción de individuos que perciben prestación por desempleo en cada grupo ( $\mathrm{p}$-lim=0,239). Los que perciben prestaciones inferiores pertenecen en mayor proporción al colectivo de egresados 2003/04, en tanto que las prestaciones inferiores a 12.0000 son proporcionalmente mayores entre los que finalizaron en 2001/02. 
Por ultimo se analizaron las expectativas para encontrar empleo. En relación con este aspecto, los egresados en 2001/02 tienen mayores expectativas a corto plazo ("bastantes" un $40 \%$ y "alguna" un 28), en tanto que los graduados posteriormente indican en mayor proporción "alguna" expectativa ya sea a corto plazo (38\%) o a largo plazo (25\%).

TABLA 13. EXPECTATIVAS POR COlectivo

\begin{tabular}{|c|c|c|c|c|c|c|}
\hline EXRECTATIVAS & $\begin{array}{c}\text { Ninguna, nl a } \\
\text { corto nl a largo } \\
\text { plazo }\end{array}$ & $\begin{array}{c}\text { Alguna, a corto } \\
\text { plazo }\end{array}$ & $\begin{array}{c}\text { Alguna, a largo } \\
\text { plazo }\end{array}$ & $\begin{array}{c}\text { Bastante, a } \\
\text { corto plazo }\end{array}$ & $\begin{array}{c}\text { Bastante, a } \\
\text { largo plazo }\end{array}$ & TOTAL \\
\hline $\begin{array}{c}\text { EGRESADOS } \\
\text { 2001/02 }\end{array}$ & $7,32 \%$ & $27,80 \%$ & $18,05 \%$ & $40,49 \%$ & $6,34 \%$ & $100,0 \%$ \\
\hline $\begin{array}{c}\text { EGRESADOS } \\
\text { 2003/04 }\end{array}$ & $8,57 \%$ & $38,21 \%$ & $25,00 \%$ & $16,07 \%$ & $12,14 \%$ & $100,0 \%$ \\
\hline
\end{tabular}

\section{2. EgRESAdOS INACTIVOS}

De los egresados que afirmaron no estar desempeñando una actividad remunerada en la actualidad, 243 confirman no realizar una búsqueda activa de empleo -un $16,2 \%$ del total de titulados de la Universidad de Córdoba.

De los egresados que afirmaron no estar desempeñando una actividad remunerada en la actualidad, 243 confirman no realizar una búsqueda activa de empleo -un $16,2 \%$ del total de titulados de la Universidad de Córdoba-. Entre promociones son los titulados en el curso 2003/04 los que aglutinan al 62.14\% del total inactivo, frente al $37,86 \%$ de los que acabaron en 2001/02.

En cuanto al sexo, la tasa de inactividad es siete puntos porcentuales superior entre población egresada femenina que la masculiná. Además, se puede observar que aproximadamente dos de cada tres titulados inactivos son mujeres.

Si se detallan los resultados para las macroáreas de conocimiento, se observa que las titulaciones asociadas a Ciencias Sociales y Jurídicas son las que mayor porcentaje de titulados inactivos presentan. Este resultado podría considerarse previsible si tenemos en cuenta que un tercio de la muestra analizada pertenece a alguna titulación de esta macroárea, con lo que los resultados en términos absolutos nos pueden inducir a apreciaciones poco rigurosas. En la tasa de inactividad se observa que son las titulaciones de Humanidades las que en mayor proporción recogen al egresado inactivo, siendo uno de cada tres egresados en humanidades individuos parados que no realizan búsqueda de empleo.

En relación al ciclo universitario se observa que las titulaciones de primer ciclo -diplomaturas o ingenierías técnicas- presentan menor tasa de inactividad, un $12 \%$, que las de titulaciones de segundo ciclo cuya tasa se eleva hasta el $18 \%$. En total la tasa de inactividad entre los titulados de la universidad de Córdoba es del 16,20\%. 
Tabla 14. Análisis de la Población inactiva

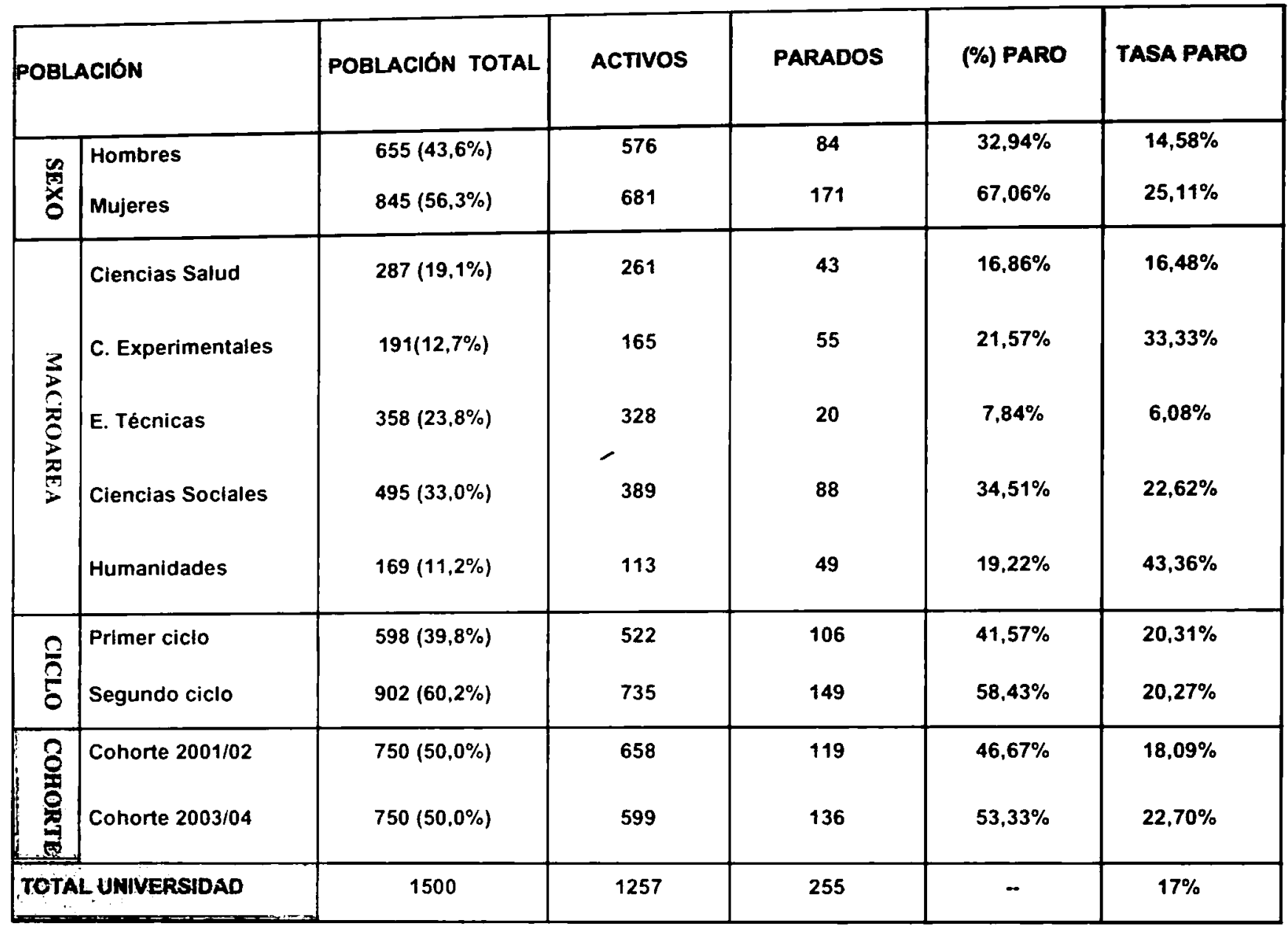

Por último, la principal razón que señalan los universitarios para su inactividad es la preparación de oposiciones, en un 61.2\% de los casos; seguida de la decisión de continuar con otros estudios.

Tabla 15. Situación de los egresados inactivos por sexo

\begin{tabular}{|l|c|c|c|}
\hline \multicolumn{1}{|c|}{ SITUACION } & HOMBRE & MUJER & TOTAL \\
\hline He estado siempre desempleado/a & & $1,80 \%$ & $1,20 \%$ \\
\hline \begin{tabular}{l|c|c|} 
He tenido trabajos temporales relacionados con mis \\
He tenido trabajos temporales sin relación con mis \\
estudios
\end{tabular} & $6,40 \%$ & $3,00 \%$ & $4,10 \%$ \\
\hline He preparado oposiciones & $5,10 \%$ & $5,50 \%$ & $5,40 \%$ \\
\hline He seguido con otros estudios & $21,80 \%$ & $62,80 \%$ & $61,20 \%$ \\
\hline Otros & $9,00 \%$ & $4,90 \%$ & $6,20 \%$ \\
\hline \multicolumn{1}{|c|}{ TOTAL } & $100,00 \%$ & $100,00 \%$ & $100,00 \%$ \\
\hline
\end{tabular}


La mayoría de los encuestados opinan que están desempleados porque no se han dedicado de forma activa a buscar empleo. En torno al $20 \%$ consideran que está decisión está relacionada con la situación del mercado laboral.

Tabla 16. Situación de los Egresados Inactivos

\begin{tabular}{|l|c|c|c|}
\hline \multicolumn{1}{|c|}{ SITUACION } & HOMBRE & MUJER & TOTAL \\
\hline Situación del mercado laboral & $17,90 \%$ & $20,70 \%$ & $19,80 \%$ \\
\hline Deberes familiares han dificultado la búsqueda & & $3,70 \%$ & $2,50 \%$ \\
\hline Titulación inadecuada para los trabajos que se ofrecen & $7,70 \%$ & $2,40 \%$ & $4,10 \%$ \\
\hline $\begin{array}{l}\text { Carezco de formación adicional para los trabajos que se } \\
\text { ofrecen }\end{array}$ & $1,30 \%$ & & $0,40 \%$ \\
\hline Los empleos ofrecidos no cumplian mis expectativas & $1,30 \%$ & $1,20 \%$ & $1,20 \%$ \\
\hline No me he dedicado de forma activa a buscar empleo & $53,80 \%$ & $60,40 \%$ & $58,30 \%$ \\
\hline Otros & $17,90 \%$ & $11,60 \%$ & $13,60 \%$ \\
\hline \multicolumn{1}{|c|}{ TOTAL } & $100,00 \%$ & $100,00 \%$ & $100,00 \%$ \\
\hline
\end{tabular}

\section{CONCLUSIONES}

Respecto de las tasas de actividad, de ocupación y de desempleo de los titulados de la UCO cabe hacer dos observaciones generales de interés. En primer lugar, las importantes diferencias que se observa entre macroáreas y titulaciones una vez desagregados los datos de la población egresada tomada en consideración en la muestra (referida a los egresados de 01/02 y 03/04). En segundo lugar, no existe una relación entre el grado de feminización que ostenta la enseñanza universitaria y su inserción laboral posterior, reproduciéndose por tanto en nuestra estudio la misma vulnerabilidad que en general adolece la mujer como grupo profesional de inserción (CES de España, Informe 6/2005).

Además, se puede concluir que existen niveles diferenciados de inserción laboral en el seno del colectivo según la formación de origen y el título académico Así, el primer nivel, que correspondería con un grado bastante alto de empleabilidad, está representado en las enseñanzas técnicas. El segundo nivel, que correspondería a un grado alto de empleabilidad, está ocupado por las ciencias de la salud. En un nivel medio-alto de inserción se ubican las macroáreas relativas a las ciencias experimentales y a las ciencias sociales y jurídicas, existiendo dentro de éstas últimas títulos 
específicos que cuentan con un nivel de empleabilidad próximo a niveles óptimos (v.gr. licenciado en ciencias ambientales, psicopedagogía, relaciones laborales, o ciencias del trabajo). Por el lado contrario, y con un grado insuficiente de inserción laboral, se encuentran las humanidades, con porcentajes muy por debajo de la media general del empleo egreso.

Es interesante observar que el número de contratos aumenta en aquellas titulaciones que han sido implantadas más recientemente en nuestro campus (en concreto, ciencias del trabajo, enología, tecnología de los alimentos). Ello probablemente deba explicarse en primera instancia por constituir una novedad en el mercado de trabajo, pero es revelador de la existencia de un espacio potencial en el tejido empresarial para tales titulados.

No obstante lo anterior, es necesario reflexionar ante uno de los problemas que con mayor presencia está apareciendo en la sociedades desarrolladas en los últimos tiempos y como es el desajuste entre las exigencias o requerimientos de los puestos de trabajo y las capacidades y habilidades de los individuos que los ocupan actualmente. Teóricamente, el sistema educativo debe estar al servicio del sistema productivo. En este sentido la universidad debe formar profesionales cuya preparación académica sea, además de suficiente, de aplicación directa al puesto de trabajo. Sin embargo, la realidad actual tal y como posteriormente se reflejará en este estudio es que se está produciendo un desajuste o mismatch entre las exigencias del puesto y las capacidades del egresado.

Está claro que la transición óptima del sistema educativo al empleo exige que en un plazo suficiente los egresados ocupasen un puesto de trabajo cuyos requerimientos fuesen ajustados a su nivel de educación y de capacitación. Si los desajustes entre titulación y funciones en el puesto de trabajo es algo temporal, elegido por el trabajador de manera voluntaria como estrategia para encontrar un empleo adecuado, el estudio se focalizaría en minimizar el periodo de desajuste. El problema radica cuando es algo que ocurre de forma permanente.

En esta investigación se analizaron datos de este desajuste o subempleo, se considerando que un universitario está subempleado o sobrecualificado si posee mayor nivel de educación formal que la exigida en su puesto de trabajo. El problema se centra en concretar la forma de medición de este desajuste, que nos permita establecer un correcto análisis. Sin entrar en detalles específicos destacar que tanto en los egresados del curso 2001/02 como del curso 2003/04 se presenta un porcentaje de titulados claramente subempleados en el sentido de no necesitar título universitario para desarrollar su trabajo. Un 14,3\% de los egresados del curso 2001/02 y un 10,6\% de los egresados del curso 2003/ 04 afirman no necesitarlo. Si además se incorporan los que indican que el título le ayuda, pero igualmente alguien con menor acreditación puede realizarlo (subempleo moderado), el porcentaje para ambos cursos alcanza el 32\%. Otra dimensión de este subempleo es el análisis de la coincidencia entre el campo de ocupación y el campo de estudio. Un 
$28 \%$ de los titulados en el 2001/02 trabajan en un campo que no está relacionado con su titulación, siendo el porcentaje para los egresados del 2003/04, igual al $24,2 \%$. Tal y como ocurre con el caso anterior, se observan diferencias significativas en relación con el sexo para el colectivo más antiguo donde el porcentaje de egresadas empleadas en un campo distinto $-33,6 \%$ - es superior en más de diez puntos porcentuales al porcentaje de egresados empleados en campos distintos $-22,1 \%$.

Por último poner de manifiesto la evidente situación desfavorable de la mujer titulada en la universidad. No solo por los niveles de ocupación y desempleo obtenidos, sino por el resto de aspectos relacionados con su puesto de trabajo tales como el acceso, periodo de transición, promoción o retribución, por citar alguno de ellos.

\section{BIBLIOGRAFÍA}

ALBA RAMIREZ (1993). Mismatch in the Spanish Labor Market: Overeducation?. The Journal of Human Resources, 24. pp. 259-278.

ALBA RAMíREZ, A. (1996), En busca del primer empleo: el precio de la experiencia. Economistas, núm.70, pp:14-22

ALCAIDE, M., GONZÁLEZ, M Y FLORES I. (1996) Mercado de trabajo, reclutamiento y formación en España. Pirámide.

AlCAIDE, M., Y CEULAR, N. (2007) Temas Laborales. Revista Andaluza de la de Trabajo y Bienestar social, 88, pp:125-148.

BURKE, R. (1997) Correlates of under-employment among recent business school graduates, 18, pp: 627:633.

CARIDAD, JM (1998) Econometría: modelos econométricos y series temporales. Ed. Reverté.

CES DE ESPAÑA, Panorama sociolaboral de la mujer en España, n 46, 2006.

CEULAR, N. Y CARIDAD; JM (2007). El perfil del egresado subempleado. XVII Jornadas Hispanolusas de Gestion Cientifica.

CONGREGADO, I. Y GARCÍA, I. (2002). El problema del desempleo en la economía andaluza (1999-2001): Análisis de la transición desde la educación al mercado laboral. CENTRA., 17.

CORRALES, E. Y RODRÍGUEZ, B. (2003). La transición del sistema educativo al mercado laboral. Análisis de los factores determinantes del piimer empleo. Actas de las V Jornadas de Economía de la Educación. Universidad Rovira i Virgili. 
CYD (2006). La contribución de las Universidades españolas al desarrollo. Fundación Conocimiento y Desarrollo.

GARCÍA-SERRANO, C Y MALO M. (1996). Desajuste educativo y movilidad laboral. Revista de Economía Aplicada., 11, pp: 105-131.

GIL JURADO, J.A.(1998). Inserción laboral y desajuste educativo de los titulados universitarios. Un estudio de los titulados de la ULPGC.

INE (1993). La inserción laboral de las tituladas universitarias en el mercado de Trabajo.

JIMÉNEZ AGUILERA, J; SÁNCHEZ CAMPILLO, J. Y MONTERO GRANADOS, R. (2003) Educación Superior y Empelo: la situación de los Jóvenes titulados en Europa: la encuesta Cheers. Universidad de Granada.

NAVARRO, F. Y CEULAR, N (2007) Uńiversidad y Empleo. Instituto de Desarrollo Local del Ayuntamiento de Córdoba

RAHONA LÓPEZ, M. (2004) ¿La posesión de un título universitario facilita el acceso de los jóvenes al primer empleo? Una aproximación para el caso español. Revista del Ministerio de Trabajo y Asuntos Sociales, 61, pp.105-119.

SALAS VELASCO, M. (2003), Educación superior y Mercado de trabajo. Grupo Editorial Universitario.

VIDAL GARCÍA, J. (COORD) (2003). Métodos de análisis de la inserción laboral de los universitarios. Ministerio Educación y Cultura.Vidal, J.; López, R.; y Pérez, C. (2004) Formación y Empleo de los Titulados en la Universidad de León. Universidad de León, Oficina Evaluación de la Calidad.

WINEFIELD, A.H.,TIGGEMANN, M Y GOLNEY (1991). A longitudinal study of the psychological effects of unemployment and unsatisfactory employment on young adults. Journal of Applied Psychology, 76, pp: 424- 431. 